\title{
Performance Analysis of Joint Relay Selection and Power Allocation for Two-way Relay Channels
}

\author{
Sijia Lou ${ }^{1,2}$, Longxiang Yang ${ }^{1,2}$ \\ ${ }^{1}$ Wireless Communications Key Lab of Jiangsu Province Nanjing University of Posts and Telecommunications, Nanjing, \\ Jiangsu, P. R. China, 210003 \\ ${ }^{2}$ Wideband Wireless Communications and Sensor Networks Technology Lab Ministry of Education of China, Nanjing, Jiangsu, \\ P. R. China, 210013 \\ lousij@gmail.com,yanglx@njupt.edu.cn
}

\begin{abstract}
This paper investigated the outage probability bound of joint relay selection and power allocation for two-way relay channels (TWRC). The two transceivers exchanged information with the help of the relay during two time slots based on amplify-andforward (AF). A tight lower bound of outage probability with optimal power allocation was derived. Based on the close-form outage probability bound, we proposed a simplified relay selection criterion, which just calculated harmonic mean of instantaneous channel gains. Simulation verifies the theoretical analysis we proposed. The lower bound we proposed is tight comparing with actual performance for all signal-to-noise ratio (SNR) range, meanwhile more accurate than any existing bound.
\end{abstract}

Index Terms - Two-way relay, outage probability, Amplify-andForward, joint relay selection and power allocation

\section{Introduction}

Cooperative communications has been widely studied recently for its ability to enhance the throughput and reliability of wireless networks [1]. However, traditional one-way relay protocol causes an inherent spectral efficiency loss since all nodes operate under half-duplex mode. Two-way relaying is proposed to improve spectral efficiency by avoiding the $1 / 2$ pre-log factor in capacity calculating [2]. Several networkcoding schemes have been presented for two-way relay systems, such as amplify-and-forward (AF), decode-andforward (DF).

Two-way AF relaying is more practically attractive than DF relaying due to the simple processing at the relay transceiver and saving one more time slot. The two-way AF protocol takes two time slots to assist information exchanging. At the first time slot, both transceivers simultaneously transit their information symbols to the relay. At the second time slot, relay node broadcasts the mixture by amplifying and forwarding operation [3]. The received signal-to-noise ratio (SNR) expression of two-way AF relaying had been given in [4] and the achievable sum rate had been derived in [5].

Relay selection (RS) and optimal power allocation (OPA) are two main methods to improve the performance of two-way relaying system. Most of the RS schemes are considered selecting one best relay to forwarding the information by a certain criterion. Relay selection in two-way AF relaying over Rayleigh fading had been presented in [6]-[11]. A relay selection scheme was proposed for AF-based TWRC, where bounds on the sum rate, symbol error rate and outage probability were analyzed in high SNR range [6]. Closed-form expression of the outage probability was derived for analog network coding-based TWRC with single RS in [7]. The selection criterion in above two schemes was based on maximizing the minimum signal-to-noise ratio. And some other criteria such as maximum sum rate, minimum outage probability or maximum mutual information were proposed in [8]-[10].

However, transmission power is restricted in modern communications. In [11], the author limited the transmission power at each node, and maximized sum rate that the system can be obtained. A resource allocation scheme using nonlinear optimization was proposed in [12], the transmission power at each node is separately limited to less than or equal to the maximum power. In [13], author considered minimizing total power consumption on the premise of meeting requirement of transmission rate of each sub-channel. A joint scheme combined relay selection and power allocation was firstly proposed in [14], in which maximized the balanced SNR. In [15], author proposed a power allocation scheme that simultaneously minimized the outage probability and maximized the total mutual information under total power constant in a single two-way relay system. The author extended it to multi-relay scenario [16], where the relay with minimum outage probability was selected to assist information exchanging.

To the best of our knowledge, there have been few literatures on investigating outage probability performance for joint relay and optimum power allocation. The scheme in [14] increases the relay's burden on power consumption since amplifying coefficient at selected relay is not normalized. The analysis in [15] is limited to a two-way system with single relay. The lower outage bound in [16] is not ideal because the PDF approximation of SNR is unsuitable for multi-relay scenario. This has motivated our work. Outage probability calculation in this paper adopts the idea of calculus, by which the CSI interval is decomposed into small pieces, and then accurately approximation of outage probabilities were obtained one by one. We derive a tight lower bound of outage probability in closed-form, which is closer than any existing bound in all SNR range. 
The rest of this paper is organized as follows. In Section II, we present the system model. In Section III, the tight lower bound of outage probability is derived in a joint relay selection and power allocation case. Numerical simulations are presented in Section IV and Section V concludes the paper.

\section{System model}

As shown in Fig.1, the system we considered is a bidirectional network, where two transceivers tend to exchange information through one of the $\mathrm{K}$ relay nodes. We use $S_{1}$, $S_{2}$ and $R_{i}$ to denote the first transceiver, the second transceiver, and the relays, respectively. The transceivers select the relay with the best channel state information (CSI) from the candidate relays to assist information exchanging. All nodes are equipped with single antenna, and operate in a halfduplex mode. The signals are coded using binary phase shift keying modulation (BPSK) at each transceiver. For simplicity, we assume that there is no direct path between the transceivers. However, the performance bound analyzed here can be extended to the case with direct path.

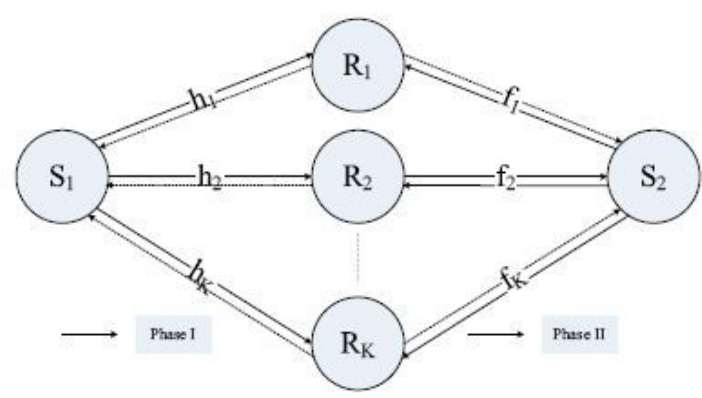

Fig. 1 System Model

Each sub-channel in this network is assumed to experience independent slow and frequency non-selective Rayleigh fading. Considering channel reciprocity, the complex channel coefficients between $S_{1}$ and $R_{i}, R_{i}$ and $S_{2}$ are denoted by $h_{i}, f_{i}$ respectively, which are complex Gaussian random variables with zero mean and variances $\mathrm{W}_{h_{i}}$ and $\mathrm{W}_{f_{i}}$. So $\left|h_{i}\right|^{2}$ and $\left|f_{i}\right|^{2}$ subject exponential distribution with parameters $l_{h_{i}}=\frac{1}{\mathrm{~W}_{h_{i}}}$ and $l_{f_{i}}=\frac{1}{\mathrm{~W}_{f_{i}}}$. The additive white Gaussian noise at $S_{1}, S_{2}$ and $R_{i}$ are assumed to be a complex Gaussian random variable with zero mean and unit variance. It is assumed that transceivers have full CSI as well as the relays to implement perfect self-interference cancelation in AF-based TWRC.

The protocol shown in Fig. 1 takes two time slots to exchange information. At the first time slot, $S_{1}$ and $S_{2}$ send signals to all candidate relay nodes. The received signal at $i$ th relay is expressed as

$$
x_{i}=\sqrt{E_{1}} h_{i} x_{1}+\sqrt{E_{2}} f_{i} x_{2}+n_{i},
$$

where $x_{1}, x_{2}$ are signals from

transceiver $S_{1}$ and $S_{2}$ respectively, $E_{1}$ and $E_{2}$ are transmit powers of $S_{1}$ and $S_{2}, n_{i}$ is Gaussian noise at relay $i$.

At the second time slot, a relay with best performance amplifies $x_{i}$ with an amplifying coefficient $r$ and transmits it to $S_{1}$ and $S_{2}$. The amplifying coefficient $r$ can be chosen as

$$
r_{i}=\sqrt{\frac{E_{r}}{E_{1}\left|h_{i}\right|^{2}+E_{2}\left|f_{i}\right|^{2}+1}} .
$$

The choice makes the analysis intractable. In the following research, it is approximated as

$$
r_{i}=\sqrt{\frac{E_{r}}{E_{1}\left|h_{i}\right|^{2}+E_{2}\left|f_{i}\right|^{2}}}
$$

which has been proved very close to the exact value for all the SNR range[17][18].

The received signal at $S_{1}$ and $S_{2}$ can be expressed as

$$
\begin{aligned}
& y_{i 1}=\sqrt{E_{1} r_{i}} h_{i}^{2} x_{1}+\sqrt{E_{2} r_{i}} h_{i} f_{i} x_{2}+r_{i} h_{i} n_{i}+n_{1} \text {, } \\
& y_{i 2}=\sqrt{E_{2}} r_{i} f_{i}^{2} x_{2}+\sqrt{E_{1}} r_{i} h_{i} f_{i} x_{1}+r_{i} f_{i} n_{i}+n_{2} \text {, }
\end{aligned}
$$

where $n_{1}, n_{2}$ denote Gaussian noise at $S_{1}$ and $S_{2}$. We can subtract the self-interference from $y_{i 1}$ and $y_{i 2}$, than obtain

$$
\begin{aligned}
& y / 9=y_{i 1}-\sqrt{E_{1}} r_{i} h_{i}^{2} x_{1}=\sqrt{E_{2}} r_{i} h_{i} f_{i} x_{2}+r_{i} h_{i} n_{i}+n_{1}, \\
& g / 9=y_{i 2}-\sqrt{E_{2}} r_{i} f_{i}^{2} x_{2}=\sqrt{E_{1}} r_{i} h_{i} f_{i} x_{1}+r_{i} f_{i} n_{i}+n_{2} .
\end{aligned}
$$

The transceivers can obtain signal symbols $x_{2}$ and $x_{1}$ by decoding the received signals $y_{i 1}$ and $y_{i 2}$.

\section{Outage probability analysis}

In this section, outage probability performance of joint relay selection and optimum power allocation scheme is analyzed. We propose a tight lower bound of outage probability of this joint scheme.

Through (6) and (7), the instantaneous SNR at $S_{1}$ and $S_{2}$ can be respectively expressed as

$$
\begin{gathered}
g_{i 1}=\frac{E_{r} E_{2}\left|h_{i}\right|^{2}\left|f_{i}\right|^{2}}{\left(E_{r}+E_{1}\right)\left|h_{i}\right|^{2}+E_{2}\left|f_{i}\right|^{2}}, \\
g_{i 2}=\frac{E_{r} E_{1}\left|h_{i}\right|^{2}\left|f_{i}\right|^{2}}{\left(E_{r}+E_{2}\right)\left|f_{i}\right|^{2}+E_{1}\left|h_{i}\right|^{2}},
\end{gathered}
$$


We define the outage probability of TWRC-AF protocol as the probability that either $I_{1}$ or $I_{2}$ is under the target rate $\mathrm{R} / 2$,

$$
P_{\text {outage }}\left(R_{i}\right)=\operatorname{Pr}\left(I_{i 1}<\frac{R}{2} \text { or } I_{i 2}<\frac{R}{2}\right),
$$

where

$$
I_{i 1}=\frac{1}{2} \log _{2}\left(1+g_{i 1}\right), I_{i 2}=\frac{1}{2} \log _{2}\left(1+g_{i 2}\right) .
$$

Pre-log factor $1 / 2$ presents information exchange takes two time slots.

Since in equal power allocation (EPA) system the power consumed is $3 \mathrm{E}\left(E_{1}=E_{2}=E_{r}=E\right)$, the joint scheme has a same total power constraint $E_{\max }=3 E$. The optimum power allocation (OPA) problem is represented as

$$
\min _{E_{1}, E_{2}, E_{r}, i}\left(P_{\text {outage }}\left(R_{i}\right)\right) \text { subject to } E_{1}+E_{2}+E_{r}=3 E,
$$

Following from (8-11), the optimization problem in (12) is equivalent to the following formulation.

$$
\max _{E_{1}, E_{2}, E_{r}} \min \left(g_{i 1}, g_{i 2}\right) \text { subject to } E_{1}+E_{2}+E_{r}=3 E .
$$

The max-min problem can be solved in the Theorem 1

$$
\begin{aligned}
P_{\text {looser }}\left(R_{i}\right)= & 1-\sum_{n=0}^{N}\left(\frac{a_{n}^{2} \lambda_{h_{i}}}{a_{n}^{2} \lambda_{h_{i}}+\lambda_{f_{i}}} \exp \left(\left(-\left(b_{n}+1\right)^{2} \lambda_{h_{i}}+\frac{\left(b_{n}+1\right)^{2}}{a_{n}^{2}} \lambda_{f_{i}}\right) \Upsilon\right)\right. \\
& -\frac{b_{n}^{2} \lambda_{h_{i}}}{b_{n}^{2} \lambda_{h_{i}}+\lambda_{f_{i}}} \exp \left(\left(-\left(b_{n}+1\right)^{2} \lambda_{h_{i}}+\frac{\left(b_{n}+1\right)^{2}}{b_{n}^{2}} \lambda_{f_{i}}\right) \Upsilon\right) \\
& +\frac{a_{n}^{2} \lambda_{f_{i}}}{\lambda_{h_{i}}+a_{n}^{2} \lambda_{f_{i}}} \exp \left(\left(-\frac{\left(b_{n}+1\right)^{2}}{a_{n}^{2}} \lambda_{h_{i}}+\left(b_{n}+1\right)^{2} \lambda_{f_{i}}\right) \Upsilon\right) \\
& \left.-\frac{b_{n}^{2} \lambda_{f_{i}}}{\lambda_{h_{i}}+b_{n}^{2} \lambda_{f_{i}}} \exp \left(\left(-\frac{\left(b_{n}+1\right)^{2}}{b_{n}^{2}} \lambda_{h_{i}}+\left(b_{n}+1\right)^{2} \lambda_{f_{i}}\right) \Upsilon\right)\right) \\
& -\frac{\lambda_{h_{i}}}{a_{N}^{2} \lambda_{h_{i}}+\lambda_{f_{i}}} \exp \left(\left(-a_{N}^{2} \lambda_{h_{i}}+\lambda_{f_{i}}\right) \Upsilon\right) \\
& -\frac{\lambda_{h_{i}}}{\lambda_{h_{i}}+a_{N}^{2} \lambda_{h_{i}}} \exp \left(\left(-\lambda_{h_{i}}+a_{N}^{2} \lambda_{f_{i}}\right) \Upsilon\right) .
\end{aligned}
$$

shown in [18].

Theorem 1: When $E_{1}+E_{2}+E_{r}=3 E$, the optimum power allocation that minimizes the outage probability $P_{\text {outage }}\left(R_{i}\right)$ of the $\mathrm{AF}$ protocol is given by

$$
\begin{aligned}
& E_{1}=\frac{3\left|f_{i}\right|}{2\left(\left|h_{i}\right|+\left|f_{i}\right|\right)} E, \\
& E_{2}=\frac{3\left|h_{i}\right|}{2\left(\left|h_{i}\right|+\left|f_{i}\right|\right)} E,
\end{aligned}
$$

$$
E_{r}=\frac{3}{2} E
$$

The optimal scheme allocates more power to the weaker traffic flow for lower outage probability. With the allocation factors, the SNR in (8) (9) can be expressed as

$$
\gamma_{i 1}=\gamma_{i 2}=\frac{3 E}{2}\left(\frac{\left|h_{i}\right|\left|f_{i}\right|}{\left|h_{i}\right|+\left|f_{i}\right|}\right)^{2} \text {. }
$$

Furthermore, the lower bound of outage probability with the help of $i$ th relay can be expressed as (14) at the top of this page, where

$$
a_{n}=1+(n+1) \mathrm{D}, b_{n}=1+n \mathrm{D}, ? \quad \frac{2\left(2^{R}-1\right)}{3 E} .
$$

\section{Proof: See Appendix A}

The values of $\mathrm{D}$ and $\mathrm{N}$ decide the accuracy of outage probability bound. When $\mathrm{D}=1$ and $\mathrm{N}$ ? 1 , (25) can be approximated as $\frac{\left|h_{i}\right|\left|f_{i}\right|}{\left|h_{i}\right|+\left|f_{i}\right|} » \frac{\left|h_{i}\right|}{(2+n \mathrm{D})}, P_{\text {lower }}\left(R_{i}\right)$ becames extremely tight.

Then we can obtain lower bound of outage probability of joint relay selection and power allocation by

$$
P_{\text {outage }}(R)=\prod_{i=1}^{K} P_{\text {outage }}\left(R_{i}\right)>\prod_{i=1}^{K} P_{\text {loverer }}\left(R_{i}\right),
$$

the relay selection problem can be expressed as

$$
k=\arg \min _{i}\left(P_{\text {outage }}\left(R_{i}\right)\right) .
$$

Since the monotonically increasing function (11) and equation (15), the relay selection problem can be simplified as follows

$$
k=\arg \max _{i}\left(\frac{\left|h_{i}\right|\left|f_{i}\right|}{\left|h_{i}\right|+\left|f_{i}\right|}\right) .
$$

The above criterions can instead the criterion in [19] for less computation complexity. Therefore, the relay selection process can be described as follows. The transceivers, which know all CSIs, calculate harmonic mean of $\left|h_{i}\right|$ and $\left|f_{i}\right|$ of each relay, select the relay with maximum harmonic mean, and then broadcast the index to all relays.

\section{Numerical results}

In this section, we verify analysis results and compare with other algorithm by simulation. The TWRC system is assumed having 2 relay nodes, and the target rate $\mathrm{R}$ is $1 \mathrm{bps} / \mathrm{Hz}$.

First, we assume the channel is a symmetric channel, the expected values of channel gain are $\mathrm{W}_{h_{i}}=1, \mathrm{~W}_{f_{i}}=1$, for $\mathrm{i}=1,2$. We observe the gap between the exact value and theoretical value given by (14) with different $\mathrm{N}$ and D . Fig.2 shows lower bound of outage probability against SNR with (i) $\mathrm{N}=2, \mathrm{D}=1$ (ii) $\mathrm{N}=10000, \mathrm{D}=0.01$. As shown in the figure, 
the lower bound of traditional RS-EPA scheme is tight. However, the SNR estimation method in RS-EPA scheme is unsuitable for that in RS-OPA scheme at low SNR region. At outage probability of $10^{-1}$, the bound given in [16] has $5 \mathrm{db}$ difference with actual performance.

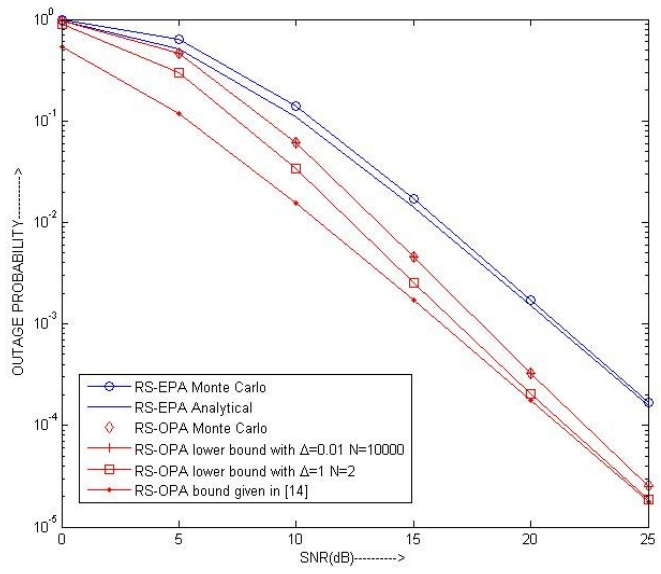

Fig.2 symmetric system with $\mathrm{W}_{h_{i}}=1, \mathrm{~W}_{f_{i}}=1(i=1,2)$

Meanwhile, for all SNR region, the bound we derived in section III is almost overlap over actual result when $\mathrm{N}=10000, \mathrm{D}=0.01$ and a fixed $1 \mathrm{~dB}$ gap with actual result when $\mathrm{N}=2, \mathrm{D}=1$, which is also more accurate than the existing bound. The simulation with asymmetric channel state is showed in fig. 3, which also confirms the conclusions above.

$$
\begin{aligned}
& \operatorname{Pr}_{1}(n)=\operatorname{Pr}\left(\frac{\left|h_{i}\right|\left|f_{i}\right|}{\left(\left|h_{i}\right|-(1+n \Delta)\left|f_{i}\right|\right)+(2+n \Delta)\left|f_{i}\right|}\right)^{2} \\
& \left.>\Upsilon,(1+n \Delta)\left|f_{i}\right| \leq\left|h_{i}\right| \leq(1+(n+1) \Delta)\left|f_{i}\right|\right) \mid \\
& \left.<\tilde{\operatorname{Pr}}_{\mathrm{r}_{1}}(n)=\operatorname{Pr}\left(\left(\frac{\left|h_{i}\right|}{(2+n \Delta)}\right)^{2}>\Upsilon,(1+n \Delta)\left|f_{i}\right| \leq\left|h_{i}\right| \leq(1+(n+1) \Delta)\left|f_{i}\right|\right)\right) \\
& =\operatorname{Pr}\left(X>\left(1+b_{n}\right)^{2} \Upsilon, b_{n}^{2} Y \leq X \leq a_{n}^{2} Y\right) \\
& =\operatorname{Pr}\left(Y>\frac{\left(1+b_{n}\right)^{2}}{b_{n}^{2}} \Upsilon, b_{n}^{2} Y \leq X \leq a_{n}^{2} Y\right) \\
& +\operatorname{Pr}\left(\frac{\left(1+b_{n}\right)^{2}}{a_{n}^{2}} \Upsilon<Y<\frac{\left(1+b_{n}\right)^{2}}{b_{n}^{2}} \Upsilon, b_{n}^{2} Y \leq X \leq a_{n}^{2} Y\right) \\
& =\frac{a_{n}^{2} \lambda_{h_{i}}}{a_{n}^{2} \lambda_{h_{i}}+\lambda_{f_{i}}} \exp \left(\left(-\left(b_{n}+1\right)^{2} \lambda_{h_{i}}+\frac{\left(b_{n}+1\right)^{2}}{a_{n}^{2}} \lambda_{f_{i}}\right) \Upsilon\right) \\
& -\frac{b_{n}^{2} \lambda_{h_{i}}}{b_{n}^{2} \lambda_{h_{i}}+\lambda_{f_{i}}} \exp \left(\left(-\left(b_{n}+1\right)^{2} \lambda_{h_{i}}+\frac{\left(b_{n}+1\right)^{2}}{b_{n}^{2}} \lambda_{f_{i}}\right) \Upsilon\right)
\end{aligned}
$$

$$
\begin{gathered}
\tilde{\operatorname{Pr}}_{2}(n)<\operatorname{Pr}_{2}(n)=\frac{a_{n}^{2} \lambda_{f_{i}}}{\lambda_{h_{1}}+a_{n}^{2} \lambda_{f_{i}}} \exp \left(\left(-\frac{\left(b_{n}+1\right)^{2}}{a_{n}^{2}} \lambda_{h_{i}}\right.\right. \\
\left.\left.+\left(b_{n}+1\right)^{2} \lambda_{f_{i}}\right) \Upsilon\right)-\frac{b_{n}^{2} \lambda_{f_{i}}}{\lambda_{h_{1}}+b_{n}^{2} \lambda_{f_{i}}} \exp \left(\left(-\frac{\left(b_{n}+1\right)^{2}}{b_{n}^{2}} \lambda_{h_{i}}\right.\right. \\
\left.\left.+\left(b_{n}+1\right)^{2} \lambda_{f_{i}}\right) \Upsilon\right) \\
\operatorname{Pr}_{8}<\tilde{\operatorname{Pr}}_{8}=\operatorname{Pr}\left(Y>\Upsilon, a_{N}^{2} Y<X\right) \\
=\frac{\lambda_{f_{i}}}{a_{N}^{2} \lambda_{h_{i}}+\lambda_{f_{i}}} \exp \left(\left(-a_{N}^{2} \lambda_{h_{i}}+\lambda_{f_{i}}\right) \Upsilon\right)
\end{gathered}
$$

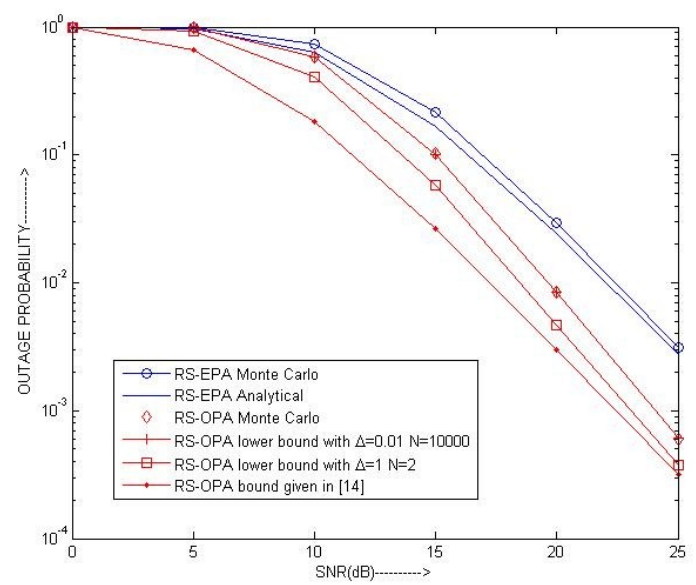

Fig. 3 asymmetric system with

$$
\mathrm{W}_{h_{1}}=1 / 8, \mathrm{~W}_{h_{2}}=1 / 2, \mathrm{~W}_{f_{1}}=1 / 4, \mathrm{~W}_{f_{2}}=1 / 4
$$

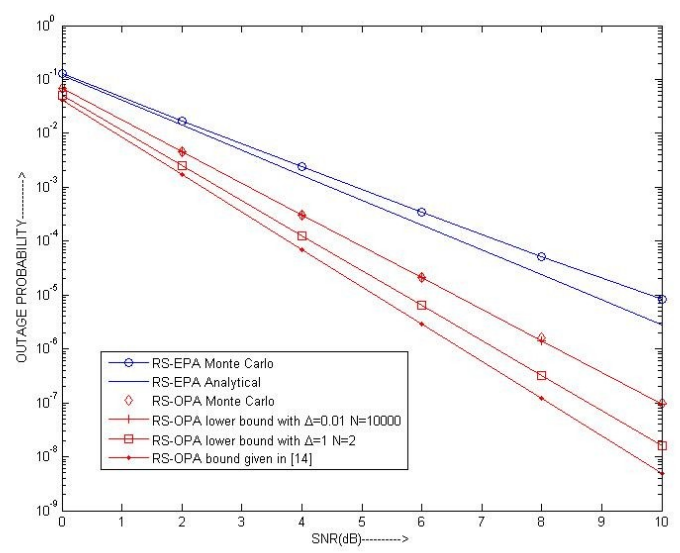

Fig. 4 outage probability against relay number $\mathrm{K}$

Fig.4 shows the outage probabilities as a function of relay number $\mathrm{K}$ with $\mathrm{SNR}=15 \mathrm{~dB}$. The gaps between Monte Carlo results and theoretic results become larger by relay number increasing. However, the lower bound we proposed is still tight, and is scarcely affected by the number of relays. 


\section{Conclusions}

In this paper, we studied outage probability performance in two-way relay networks consisting of two transceivers and multiple relay nodes. We derived a tight bound of outage probability for joint relay selection and power allocation scheme in close-form. The bound was proved exactly fitting the real results obtained by simulation at all SNR range. We also proposed a simplified criterion which used the harmonic mean of the channel gains between relays and transceivers to select the best relay. The next study is to simplify the formula proposed in this paper, derive exact outage probability expressions.

\section{Appendix A:}

$$
\begin{aligned}
P_{\text {outage }}\left(R_{i}\right) & =\operatorname{Pr}\left(I_{i 1}<\frac{R}{2} \text { or } I_{i 2}<\frac{R}{2}\right) \\
& =1-\operatorname{Pr}\left(I_{i 1}>\frac{R}{2}, I_{i 2}>\frac{R}{2}\right) \\
& \left.=1-\operatorname{Pr}\left(\frac{\left|h_{i}\right|\left|f_{i}\right|}{\left|h_{i}\right|+\left|f_{i}\right|}\right)^{2}>\Upsilon\right),
\end{aligned}
$$

where $\Upsilon=\frac{2\left(2^{R}-1\right)}{3 E}$

$$
\begin{gathered}
\left.\operatorname{Pr}\left(\frac{\left|h_{i}\right|\left|f_{i}\right|}{\left|h_{i}\right|+\left|f_{i}\right|}\right)^{2}>\Upsilon\right) \\
=\sum_{n=0}^{N} \underbrace{\left(\operatorname{Pr}\left(\mid \frac{\left|h_{i}\right|\left|f_{i}\right|}{\left|h_{i}\right|+\left|f_{i}\right|}\right)^{2}>\Upsilon,(1+n \Delta)\left|f_{i}\right| \leq\left|h_{i}\right| \leq(1+(n+1) \Delta)\left|f_{i}\right|\right)}_{\operatorname{Pr}_{1}(n)}
\end{gathered}
$$

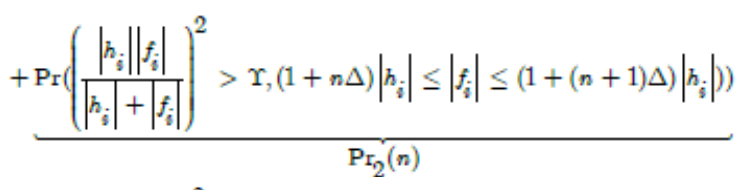$$
\underbrace{\left.+\operatorname{Pr}\left(\frac{\left|h_{i}\right|\left|f_{i}\right|}{\left|h_{i}\right|+\left|f_{i}\right|}\right)^{2}>\Upsilon,(1+(N+1) \Delta)\left|f_{i}\right| \leq\left|h_{i}\right|\right)}_{\operatorname{Pr}_{3}}
$$$$
\underbrace{\left.\operatorname{Pr}\left(\frac{\left|h_{i}\right|\left|f_{i}\right|}{\left|h_{i}\right|+\left|f_{i}\right|}\right)^{2}>\Upsilon,(1+(N+1) \Delta)\left|h_{i}\right| \leq\left|f_{i}\right|\right)}_{\operatorname{Pr}_{4}} .
$$

Let $X=\left|h_{i}\right|^{2}, Y=\left|f_{i}\right|^{2}, \mathrm{X}$ and $\mathrm{Y}$ are exponential random variables with parameters $l_{h_{i}}=\frac{1}{\mathrm{~W}_{h_{i}}}, l_{f_{i}}=\frac{1}{\mathrm{~W}_{f_{i}}}, " \mathrm{D}>0$, we have

$$
\begin{array}{r}
\frac{\left|h_{i}\right|\left|f_{i}\right|}{\left|h_{i}\right|+\left|f_{i}\right|}=\frac{\left|h_{i}\right|\left|f_{i}\right|}{\left(\left|h_{i}\right|-(1+n \mathrm{D})\left|f_{i}\right|\right)+(2+n \mathrm{D})\left|f_{i}\right|} \\
<\frac{\left|h_{i}\right|\left|f_{i}\right|}{(2+n \Delta)\left|f_{i}\right|}=\frac{\left|h_{i}\right|}{(2+n \Delta)} .
\end{array}
$$

$\operatorname{Pr}_{1}(n)$ can be calculated as shown (20) on top of previous page. Where $a_{n}=1+(n+1) \mathrm{D}, b_{n}=1+n \mathrm{D}$.

Similarly, the second probability can be evaluated in (21). When $l_{h_{i}}=\frac{1}{\mathrm{~W}_{h_{i}}}, \frac{\left|h_{i}\right|\left|f_{i}\right|}{\left|h_{i}\right|+\left|f_{i}\right|}<\frac{\left|h_{i}\right|\left|f_{i}\right|}{\left|h_{i}\right|}=\left|f_{i}\right|, \operatorname{Pr}_{3}$ can be calculated as (22). Similarly, we obtain lower bound of $\mathrm{Pr}_{4}$. In summary, the outage probability can be bounded as

$$
P_{\text {outage }}\left(R_{i}\right)>1-\left(\sum_{n=0}^{N}\left(\tilde{\mathrm{P}}_{\mathrm{r}_{1}}(n)+\tilde{\mathrm{P}}_{2}(n)\right)+\tilde{\mathrm{P}}_{\mathrm{r}_{8}}+\tilde{\mathrm{P}}_{\mathrm{r}_{4}}\right) \text {. }
$$

\section{Acknowledgment}

This work is supported by the National 973 Projects (2013CB329104), National Natural Science Foundations of China (No. 61071090, No. 61171093), National Science and Technology Key Projects (2011ZX03005-004-003), Jiangsu 973 Projects (BK2011027), Jiangsu Province Natural Science Foundation Key Projects (11KJA510001) and Postgraduate Innovation Programs of Jiangsu Scientific Research (CXLX11_0384, CXLX11_0404).

\section{Reference:}

[1] A. Sendonaris, E. Erkip, and B. Aazhang, "User cooperation diversitypart I: system description," IEEE Trans. Commun., vol. 51, no. 11, pp. 19271938, Nov. 2003.

[2] B.Rankov and A.Wittneben, "Spectral efficient protocols for half-duplex fading relay channels," IEEE J. Sel. Areas Commun., vol. 25, no. 2, pp. 379-389, 2007.

[3] S. Katti, S. Gollakota, and D. Katabi, "Embracing wireless interference: analog network coding," in Proc. ACM Special Interest Group on Data Comm. (SIGCOMM'07), Kyoto, Japan, pp. 397-408, Sep. 2007.

[4] P.Popovski and H. Yomo, "Wireless network coding by amplify-andforward for bi-directional traffic flows", IEEE Communications Letters, Vol. 11, pp. 16-18, 2007.

[5] A. Rankov and A. Wittneben, "Spectral efficient protocols for half-duplex fading relay channels", IEEE Journal on Selected Areas in Communications, Vol. 25, pp. 379-389, Feb. 2007.

[6] A. Bletsas, A. Khisti, D. P. Reed, and A. Lippman, "A simple cooperative diversity method based on network path selection," IEEE J. Select. Areas. Commun., vol. 24, no. 3, pp. 659-672, Mar. 2006

[7] K.-S. Hwang, Y.-C. Ko, and M.-S. Alouini, "Performance bounds for twoway amplify-and-forward relaying based on relay path selection,"'in Proc. 2009 IEEE Vehicular Technology Conference - Spring, pp. 1-5.

[8] P. K. Upadhyah and S. Prakriya, "Performance Bounds for Analog Network Coding Based Two-Way Relaying with Multiuser Selection Diversity", Wireless Communications and Networking Conference (WCNC), , pp. 333-338, March, 2011.

[9] C. Peng, Q. Zhang, M. Zhao, and Y. Yao, "SNCC: a selective network coded cooperation scheme in wireless networks," in Proc. IEEE ICC 2007, pp. 4219-4224, June. 2007.

[10] A. Hyadi, M. Benjillali and M. S. Alouini, "Outage Performance of Two-Way DF Relaying Systems with a New Relay Selection Metric”, 
Wireless Communications and Networking Conference (WCNC), pp.570-574, Apr. 2012.

[11] I. Ahmed, A. Nasri, and R. K. Mallik, "Relay Subset Selection and Fair Power Allocation for Best and Partial Relay Selection in Generic Noise and Interference", IEEE Transactions on Wireless Communications, Vol.11, pp. 1828-1839, May. 2012.

[12] B. Han, W. Wang and M. Peng, "Optimal Resource Allocation for Network Coding in Multiple Two-Way Relay OFDM Systems", Wireless Communications and Networking Conference (WCNC), pp. 1642-1647, Apr. 2012.

[13] M. Zhou, Q. Cui, R. Jantti and X. Tao, "Energy-Efficient Relay Selection and Power Allocation for Two-Way Relay Channel with Analog Network Coding", IEEE Communications Letters, Vol. 16, pp. 816-819, June, 2012.

[14] S. Talwar, Y. Jing, and S. Shahbazpanahi, "Joint relay selection and power allocation for two-way relay networks," IEEE Signal Process. Letter, vol. 18, no. 2, pp. 91-94, Feb 2011.

[15] Y. Zhihang, J. MinChul, and K. Il-Min, "Outage probability and optimum power allocation for analog network coding," IEEE

Transactions on Wireless Communications, vol. 10, no. 2, pp. 407-412, 2011.

[16] Zheng Ren, Yongyu Chang, Yongliang Zhang, Dacheng Yang, “Outage probability of joint relay selection and power allocation for two-way relay networks over Rayleigh fading Channels", Vehicular Technology Conference (VTC Spring),May 2012.

[17] P. A. Anghel and M. Kaveh, "Exact symbol error probability of a cooperative network in a Rayleigh-fading environment," Wireless Commun. IEEE Trans., vol. 3, pp. 1416-1421, Sep. 2004.

[18] A. Ribeiro, X. Cai, and G. B. Giannakis, "Symbol error probabilities for general cooperative links," IEEE Trans. Wireless Commun., vol. 4, pp. 1264-1273, May 2005. 